\title{
Search for a remnant violation of the Pauli exclusion principle in a Roman lead target
}

\author{
Kristian Piscicchia ${ }^{1,2, a}{ }^{\mathbb{D}}$, Edoardo Milotti $^{3}$, Aidin Amirkhani $^{4}$, Sergio Bartalucci $^{2}$, Sergio Bertolucci $^{5}$, \\ Massimiliano Bazzi ${ }^{2}$, Mario Bragadireanu ${ }^{2,6}$, Michael Cargnelli ${ }^{7}$, Alberto Clozza $^{2}$, Raffaele Del Grande ${ }^{1,2, b}$, \\ Luca De Paolis $^{2}$, Jean-Pierre Egger ${ }^{8}$, Carlo Fiorini ${ }^{4}$, Carlo Guaraldo ${ }^{2}$, Mihail Iliescu ${ }^{2}$, Matthias Laubenstein ${ }^{9}$, \\ Johann Marton ${ }^{7}$, Marco Miliucci ${ }^{2}$, Andreas Pichler ${ }^{7}$, Dorel Pietreanu ${ }^{2,6}$, Alessandro Scordo ${ }^{2}$, Hexi Shi ${ }^{10}$, \\ Diana Laura Sirghi ${ }^{2,6}$, Florin Sirghi $^{2,6}$, Laura Sperandio ${ }^{2}$, Oton Vazquez Doce ${ }^{11}$, Johann Zmeskal ${ }^{7}$, \\ Catalina Curceanu ${ }^{1,2,6}$ \\ ${ }^{1}$ Centro Fermi-Museo Storico della Fisica e Centro Studi e Ricerche "Enrico Fermi”, 00184 Rome, Italy \\ 2 INFN-Laboratori Nazionali di Frascati, Via Enrico Fermi 40, 00044 Frascati, Italy \\ ${ }^{3}$ Dipartimento di Fisica, Università di Trieste and INFN-Sezione di Trieste, Trieste, Italy \\ ${ }^{4}$ Politecnico di Milano, Dipartimento di Elettronica, Informazione e Bioingegneria and INFN Sezione di Milano, Milan, Italy \\ ${ }^{5}$ Dipartimento di Fisica e Astronomia, Università di Bologna, Bologna, Italy \\ ${ }^{6}$ IFIN-HH, Institutul National pentru Fizica si Inginerie Nucleara Horia Hulubei, Magurele, Romania \\ ${ }^{7}$ Stefan-Meyer-Institute for subatomic physics, Austrian Academy of Science, Wien, Austria \\ ${ }^{8}$ Institut de Physique, Université de Neuchâtel, Neuchâtel, Switzerland \\ ${ }^{9}$ Laboratori Nazionali del Gran Sasso, INFN, L'Aquila, Italy \\ ${ }^{10}$ Institut für Hochenergiephysik der Österreichischen Akademie der Wissenschaften, Wien, Austria \\ ${ }^{11}$ Excellence Cluster "Origin and Structure of the Universe", 85748 Garching, Germany
}

Received: 4 November 2019 / Accepted: 10 May 2020 / Published online: 6 June 2020

(C) The Author(s) 2020

\begin{abstract}
In this paper we report on the results of two analyses of the data taken with a dedicated VIP-Lead experiment at the Gran Sasso National Laboratory of the INFN. We use measurements taken in an environment that is especially well screened from cosmic rays, with a metal target made of "Roman lead" which is characterised by a low level of intrinsic radioactivity. The analyses lead to an improvement, on the upper bounds of the Pauli Exclusion Principle violation for electrons, which is more than one (four) orders of magnitude, when the electron-atom interactions are described in terms of scatterings (or close encounters) respectively.
\end{abstract}

\section{Introduction}

The Pauli exclusion principle (PEP) is a direct consequence of the spin-statistics connection which was first formulated by Fierz [1] and then comprehensively demonstrated by Pauli [2] using the assumptions that lay at the basis of Quantum Field Theory (QFT). Therefore, experimental tests of PEP can be viewed either as tests of the fermionic/bosonic nature

\footnotetext{
a e-mail: kristian.piscicchia@cref.it

b e-mail: raffaele.delgrande@lnf.infn.it (corresponding author)
}

of the elementary particles, or as tests of the foundations of QFT.

Experimental tests of the spin-statistics connection are complicated by a simple but stringent condition. The identity of the particles under test implies a symmetric Hamiltonian, which by its nature, cannot change the symmetry of any multiparticle wave function. Therefore, the symmetry of multiparticle states must be conserved. Moreover transitions between states with different symmetry are forbidden even in a world where the spin-statistics connection does not hold. This rule goes under the name of "Messiah-Greenberg" superselection rule [3], and it can be violated only in open quantum systems, for instance, by looking for transitions among violating states of a prepared system after the introduction of particles from outside of the considered system. If charged particles couple universally to the electromagnetic field then transitions among anomalous states occur at the standard rate. A prototype experiment of this class has been carried out in 1990 by Ramberg and Snow [4] following a suggestion of Greenberg and Mohapatra [5]. The VIP collaboration is performing high precision tests of the PEP for electrons [6-8] at the Gran Sasso National Laboratory (LNGS) of INFN exploiting this method, which consists in measuring the X-ray emission from a copper strip where a (Direct Cur- 
rent) DC current is circulated, and looking for a difference in the X-ray emission with current on and off. The basic concept is that some of the new electrons injected into the copper strip may form a wrong symmetry state with the electrons in the inner shells of the copper atoms, so that they could be radiatively captured by these atoms and emit anomalous atomic Xrays as they cascade to the fundamental level of non-Paulian atoms. The current-on state provides the signal while the current-off state provides the estimate of the background.

A careful analysis of the method immediately points to two related questions: how does one define a "new" electron? Why, besides the "new" electrons injected in the strip, should not also the electrons already present in the copper strip contribute to the signal? The problem has been first considered by Elliot et al. [9], where various levels of "newness" for the fermion-system were classified. According to [9] the free electrons present in the conduction band of the conductor will rarely interact with a specific atom. Considering the average scattering time $\left(\tau_{S}\right)$ in copper (in parenthesis values for lead), obtained from the theory of conduction in metal, $\tau_{S} \approx 2.5 \times 10^{-14} \mathrm{~s}\left(\tau_{S} \approx 1.3 \times 10^{-15} \mathrm{~s}\right.$ see [10]), one finds about $1.21 \times 10^{21}\left(2.42 \times 10^{22}\right)$ scatterings per year and, therefore, it would take at least 480 years ( 25 years) for a single electron to scatter off all the atoms in one mole of copper (lead). In [9] it is then assumed that also the free electrons already present in a conductor target can produce an anomalous X-ray signal from PEP violating transitions. In [9] two searches for PEP violating signals in lead were performed: one considering only the newly injected electrons through a DC current, the second analysing the same data considering all the electrons in the conduction band as test fermions.

In Sect. 4.1 we estimate the time dependence of the anomalous X-ray emission process, caused by atomic transitions among "wrong" symmetry states performed by electrons in the conduction band of a conductor material target. We note that if PEP violation depends on a property of a selected set of electrons, then it is not possible to detect it by searching for anomalous transitions of the conduction electrons. This is due to the fact that, although interactions are rare, since the formation of a given system of atoms belonging to a target all the possible violating transitions must already have occurred at the time of the observation; those electrons must have already been captured and reside now in stable non-Paulian atoms. On the other hand if the PEP violation is due to the wrong pairing of identical fermion pairs (see, e.g., [11]), then violating transitions of conduction electrons can occur. In this case the global wave function of the identical fermions is symmetric under the exchange of those particular pairs only. As a consequence, if the conductor target was recently produced by melting various original pieces, the electrons of one piece can interact with the atoms of another piece, thus testing the symmetry state of a new pool of electron pairs.
As pointed out in Sect. 4 the electron-atom interaction time calculation must also be revised. The view based on electron-atom scattering is useful in the context of electrical conductivity and is appealing to particle physicists who can map well-known concepts onto it, however the scatterings of conduction theory are not actually related with the atoms themselves, but depend on impurities, lattice imperfections and on phonons (see, e.g., [12]). For this reason we have criticised this view and replaced it with a different picture: in Milotti et al. [10] we propose to use the closest approaches to atoms instead of scatterings, and we estimate a "time between close encounters" which is considerably shorter, $\tau_{E} \approx 3.3 \times 10^{-17} \mathrm{~s}$ in copper $\left(\tau_{E} \approx 2.5 \times 10^{-17} \mathrm{~s}\right.$ in lead). This corresponds to about $9.5 \times 10^{23}$ close encounters per year in one mole of copper $\left(1.3 \times 10^{24}\right.$ in one mole of lead) and, consequently, it means that in less than one year one electron would have the chance to approach all the atoms in one mole of copper or lead.

In this framework we carried out a dedicated experiment using a recently forged block of Roman lead as target material, corresponding to 108 moles, surrounding a High Purity Germanium detector (HPGe). The special quality that sets Roman lead apart is that it has been long screened from cosmic radiation $[13,14]$, and for this reason it contains a low level of radionuclides. The experimental setup is especially designed for the search of new, rare events (of the order of few counts per month or even year $[15,16]$ ) by combining the low intrinsic radioactivity of the lead sample with the low cosmic ray background in the LNGS. As a consequence we obtain an improvement of a factor 16 on the upper bound of the $\beta^{2} / 2$ parameter (commonly used to quantify the probability of the PEP violation, see Sect. 2) with respect to the result presented in [9], when we adopt the same scattering scheme. The improvement on the $\beta^{2} / 2$ upper bound increases by more then four orders of magnitude when we use the "close encounters" approach. In both cases, the upper bounds obtained in this work represent the best limit ever on the PEP violation probability for electrons, when the symmetry violation is associated to the wrong pairing of electronelectron pairs.

\section{Non-Paulian transitions}

The Pauli Exclusion Principle is so deeply embedded in our theories of the microscopic world that it is extremely difficult to cast an experiment into a clear theoretical framework. Moreover, the Principle obviously stands on a solid ground, because so many features of our world appear to be robust consequences of the validity of the Principle, like, for instance, the stability of ordinary matter $[17,18]$.

Many scientists have tried to devise schemes that violate the statistics of identical particles. The case of "slightly non- 
identical" particles was discussed by Fermi as early as 1934 (see Ref. [19], the translation of the paper can be found in Ref. [20]), and he pointed out that even a tiny non-identity of electrons would dramatically change the properties of atoms during the billion of years of their existence, and would show up somehow in our world. The many difficulties that tests of the Pauli Exclusion Principle face were summarised in 1980 by Amado and Primakoff [21], who noted, for example, that if there were two different types of electrons, they would appreciably modify the well-understood pair production processes (like the Bhabha scattering in electron-positron colliders) and lead to easily measurable consequences.

In Gentile [22] suggested an intermediate statistics in which $n$ particles at most could occupy a quantum state, with the limit cases $n=1$ and $n=\infty$ for which the Fermi and Bose statistics are recovered respectively. Such a condition for the intermediate statistics does not however define a proper quantum statistics since is not invariant under change of basis (see Ref. [23]). Green [24] developed the first proper quantum statistical generalisation of the Bose and Fermi statistics. He found a trilinear commutation relation for the annihilation and creation operators, admitting an infinite set of solutions, one for each positive integer $p . p$ is the order of the parastatistics (see Ref. [25]), representing for parabosons the maximum number of particles that can occupy an antisymmetric state, while for parafermions the maximum number of particles that can occupy a symmetric state (in particular the same state). Such a parastatistics provides a set of orthodox theories but, since for example a parafermi statistics of order 2 allows up to two particles in each quantum state, the model predicts macroscopic deviations from the experimental evidences.

Ignatiev and Kuzmin presented a model consisting in a deformation of the standard Fermi oscillator [26,27]. According to this approach (also discussed by Okun' [28]) in addition to the two standard levels of a Fermi oscillator, there is an additional level that can only be accessed with a small probability $\beta^{2} / 2$. Soon after the model was proposed, Govorkov showed that it could not be generalised to a true relativistic quantum field theory, because it would lead to states with negative norm [29]. Then Greenberg put forward the "quon model" [5,30], named after the $q$ parameter introduced in the algebra which characterises the model: $a_{k} a_{l}^{\dagger}-q a_{l}^{\dagger} a_{k}=\delta_{k, l}$. The $q$ parameter is closely related to the violation probability of the Igniatiev and Kuzmin model, one can establish a correspondence and it can be shown that $\beta^{2}=1+q$. Greenberg et al. [5] were successful in obtaining a quantum field theory, but eventually it was shown, once again by Govorkov [31], that the quon field theory is not relativistic. In theories of statistics violation like the Igniatiev-Kuzmin or quon models, the violation of PEP is related to a global property of the selected set of electrons. In Sect. 4.1 we will show that this kind of models cannot be tested in an experiment searching for non-Paulian atomic transitions of the conduction electrons.

In 1988, Rahal and Campa [11] presented a different approach. They looked for possible deviations in the thermodynamic properties of matter due to the introduction of a small violation of the PEP. A small violation would subtly change the thermodynamic equilibrium of the electron gas. As it is well known a symmetry violation cannot be introduced by adding a violating term to the Hamiltonian, since any Hamiltonian (and in general any observable) describing a system of $n$ identical particles must be totally symmetric, and then permutationally invariant, with respect to all the particles. The theoretical argument as set out in [11] can be exemplified by considering a system of electrons with a wave function where a few pairs of electrons are in the same state with a small probability (obviously this holds in the framework of standard quantum mechanics and the appropriate formalism would be in terms of the density matrix). The symmetry properties of the wave function are described in terms of irreducible representations of the symmetry group of the electron system, represented by means of a Young tableau in which the wave function is symmetrised in the variables of each row and anti-symmetrised in the variables of each column. The thermodynamic properties of a free-electron gas in a metal (grand-partition function) can be obtained as well.

In simple terms let us assume that the global wave function of all the electrons in the universe does not have the exact symmetry predicted by the Principle, but contains a number of "wrong" signs. For instance, with three electrons, one such "wrong" wave function would be

$$
\begin{aligned}
\Psi\left(\mathbf{x}_{1}, \mathbf{x}_{2}, \mathbf{x}_{3}\right)=\mid & \left.\Psi_{1}\left(\mathbf{x}_{1}\right), \Psi_{2}\left(\mathbf{x}_{2}\right), \Psi_{3}\left(\mathbf{x}_{3}\right)\right\rangle \\
& +\left|\Psi_{1}\left(\mathbf{x}_{1}\right), \Psi_{3}\left(\mathbf{x}_{2}\right), \Psi_{2}\left(\mathbf{x}_{3}\right)\right\rangle \\
& +\left|\Psi_{3}\left(\mathbf{x}_{1}\right), \Psi_{1}\left(\mathbf{x}_{2}\right), \Psi_{2}\left(\mathbf{x}_{3}\right)\right\rangle \\
& +\left|\Psi_{3}\left(\mathbf{x}_{1}\right), \Psi_{2}\left(\mathbf{x}_{2}\right), \Psi_{1}\left(\mathbf{x}_{3}\right)\right\rangle \\
& -\left|\Psi_{2}\left(\mathbf{x}_{1}\right), \Psi_{3}\left(\mathbf{x}_{2}\right), \Psi_{1}\left(\mathbf{x}_{3}\right)\right\rangle \\
& -\left|\Psi_{2}\left(\mathbf{x}_{1}\right), \Psi_{1}\left(\mathbf{x}_{2}\right), \Psi_{3}\left(\mathbf{x}_{3}\right)\right\rangle
\end{aligned}
$$

because of the plus sign at the beginning of the second line. In this case the Principle would mostly hold as long as the number of wrongly entangled pairs is sufficiently small, and the violation would be conserved by any Hamiltonian that is symmetrical with respect to exchanges of identical particles (particle indistinguishability would be respected). This view of the violation is a direct challenge to the spin-statistics connection [2].

We will show in Sect. 4.1 that the view expressed by Rahal and Campa can be tested with the conduction electrons which are present in a metal target, provided that it was recently moulded from various original fragments. 




Fig. 1 Schematic representation of the Ge crystal (in green) and the surrounding lead target cylindrical sections (in grey)

\section{The VIP-lead experiment}

The high purity germanium detector (HPGe) used for this measurement is a coaxial p-type detector with a Ge crystal of $8.0 \mathrm{~cm}$ diameter, and $8.0 \mathrm{~cm}$ length with an inactive layer of lithium-doped germanium of $0.075 \mathrm{~mm}$ all around the crystal. The active germanium volume of the detector is 375 $\mathrm{cm}^{3}$. The detector is surrounded by a cylindrical target of radio-pure Roman lead, which is composed of three sections, whose thickness is of the order of $5 \mathrm{~cm}$, with a total volume $V \sim 2.17 \times 10^{3} \mathrm{~cm}^{3}$. A schematic representation of the Ge crystal (in green) and the surrounding lead target cylindrical sections (in grey) is given in Fig. 1. As discussed in Sect. 4.1 the target also rejects a large part of the residual background which survives the external shielding.

The outer part of the passive shielding of the HPGe detector consists of lead $(30 \mathrm{~cm}$ from the bottom and $25 \mathrm{~cm}$ from the sides). The inner layer of the shielding $(5 \mathrm{~cm})$ is composed of electrolytic copper. The sample chamber has a volume of about $151\left((250 \times 250 \times 240) \mathrm{mm}^{3}\right)$. The shield and the cryostat are enclosed in an air-tight steel housing of $1 \mathrm{~mm}$ thickness, which is continuously flushed with boiloff nitrogen from a liquid nitrogen storage tank, in order to reduce contact with external air (which contains environmental radon) to a minimum. On the bottom and on the sides $5 \mathrm{~cm}$ thick borated polyethylene plates are placed, which give a partial reduction of the neutron flux going towards the detector. The data acquisition system is a Lynx Digital Signal Analyser controlled via personal computer software GENIE 2000, both from Canberra-Mirion. A description of a similar detector can be found in $[15,16]$. The experimental setup is
Table 1 Values of the parameters which characterise the Roman lead target

\begin{tabular}{llll}
\hline$\tau_{S}$ & $n_{e}\left(\mathrm{~m}^{-3}\right)$ & $N_{\text {free }}$ & $\Delta t / \tau_{S}$ \\
\hline $1.30 \cdot 10^{-15} \mathrm{~s}$ & $1.33 \cdot 10^{29}$ & $2.89 \cdot 10^{26}$ & $2.78 \cdot 10^{21}$ \\
\hline
\end{tabular}

operated in the low background environment of the LNGS (INFN).

The whole detector is characterised and all of its components have been put into a validated Monte Carlo (MC) code (Ref. [32]) based on the GEANT4 software library (Ref. [33]). This allowed determining, via MC simulation, the efficiency for the detection of photons emitted inside the $\mathrm{Pb}$ target.

The data analysed in this work correspond to a total acquisition time $\Delta t \approx 42 \mathrm{~d} \approx 3.6 \cdot 10^{6} \mathrm{~s}$, collected in summer 2016 . The parameters relevant to this paper and specific of our $\mathrm{Pb}$ target are summarised in Table 1, see Sect. 4.1 for the details.

\section{Statistical capture model and data analysis}

We consider a block of metal which may contain a fraction of "anomalous" electrons. Each of them may settle in a wrong symmetry state in the fundamental state of one of the atoms. When an anomalous conduction electron meets the atom containing its wrongly paired counterpart, we expect the electron pair to settle in the lowest potential energy state. This state has two "paired electrons" and a "normal electron" in the innermost atomic shell. This brings about a reconfiguration of the electronic structure of the lead atoms in the bulk metal, so that the external electron configuration of the new anomalous lead is close to that of thallium. As a consequence of the reduced effective charge the $\mathrm{K}_{\alpha}$ violating transitions would be shifted with respect to the standard transitions, this is the experimental signature of PEP violation we look for, the energies of the violating transitions are given in Sect. 4.2. Since the original moulding of the metal piece, some of the electrons already had the chance to perform non-Paulian transitions and hence reside in stable non-Paulian atoms at the time of the experimental observation. This fraction of possible PEP violating electrons is hidden from the observer. The potential non-Paulian transitions occur as a consequence of interactions of conduction electrons with atoms which gradually deplete the reservoir of "anomalous" electrons. The depletion process is described in Sect. 4.1.

In the model adopted in [9] the interactions correspond to the scatterings in the standard conduction picture. In [10] we consider a different approach and the condition for the free electron-atom interaction is a significant overlap of the wave functions, which we call close encounter. In both cases the mathematics describing the depletion process is the 
same, however the data analysis proceeds differently for the two scenarios as a consequence of two separate, important aspects. The first is the definition of the time between interactions (which are scatterings in [9] and are close encounters in [10]), the second is the estimate of the capture probability. For this reason - for the sake of a fair comparison with the results of [9]- we first adopt the conduction picture in Sect. 4.2. We then move to the close encounters scheme in Sect. 4.3 .

\subsection{The depletion of the reservoir of free pairs}

In the time interval $T$ any given electron experiences on average $T / \tau$ interactions with the atoms in the metal block, where $\tau$ is the mean interaction time. If there are $N$ atoms in the block then, on average, there are $T / N \tau$ interactions between a given atom and a given electron in the block. This means that for a particular electron-atom pair the probability of experiencing $k$ interactions in the time interval $T$ is

$P(k)=\frac{(T / N \tau)^{k}}{k !} \exp (-T / N \tau)$,

assuming that the interactions are independent events so that the standard hypotheses for the validity of a Poisson model hold. Then, the probability of no interaction during time $T$ is just

$p=P(0)=\exp (-T / N \tau)$,

and when interaction implies capture (as in the close encounters scheme of Ref. [10]) this is the same as the probability that an electron is not captured during time $T$. Instead when we consider a capture probability $P_{\mathrm{cpt}}<1$, i.e., whenever capture is not granted in one interaction so that multiple interactions without capture are possible (as in the scattering scheme of Ref. [9]), the probability that the electron is not captured in time $T$ is:

$$
\begin{aligned}
p= & \exp (-T / N \tau)+\left(1-P_{\mathrm{cpt}}\right) \frac{(T / N \tau)}{1 !} \exp (-T / N \tau) \\
& +\cdots+\left(1-P_{\mathrm{cpt}}\right)^{k} \frac{(T / N \tau)^{k}}{k !} \exp (-T / N \tau)+\cdots \\
= & \sum_{k=0}^{k=\infty}\left(1-P_{\mathrm{cpt}}\right)^{k} \frac{(T / N \tau)^{k}}{k !} \exp (-T / N \tau) \\
= & \exp \left[-T /\left(N \tau / P_{\mathrm{cpt}}\right)\right] .
\end{aligned}
$$

This is also the fraction of electrons not yet captured after time $T$. Therefore the total number of free "anomalous" electrons in the metal block decreases in time, and the expected number of X rays from violating PEP transitions (correspond- ing to the PEP violation probability $\beta^{2} / 2$ ) is given by:

$N_{X} \approx N_{\text {free }} N_{\text {int }} P_{\text {cpt }} \epsilon_{\text {tot }} \frac{\beta^{2}}{2} \exp \left(-T P_{\text {cpt }} / N \tau\right)$,

where $T$ is the age of the metal block, $N_{\text {int }}=\Delta t / \tau$ is the number of interactions per electron (with $\Delta t$ the measurement time), $\epsilon_{\text {tot }}$ is the detection efficiency and $N_{\text {free }}=n_{e} \times V$ is the number of conduction electrons in the target volume $V$ with electron density $n_{e}$.

We can estimate the average number of anomalous X-rays by taking $N_{X}^{m}$, the measured $\mathrm{X}$-ray excess with respect to the background level, and we obtain an estimate for the violation parameter from Eq. (5):

$\frac{\beta^{2}}{2} \exp \left(-T P_{\mathrm{cpt}} / N \tau\right) \approx \frac{N_{X}^{m}}{\epsilon_{\mathrm{tot}} P_{\mathrm{cpt}} N_{\mathrm{free}} N_{\mathrm{int}}}$.

However, the actual value of $N_{X}^{m}$ is quite small when compared with the statistical fluctuation, and in practice this equation turns into an upper bound with a definite number of standard deviations $\sigma$. For instance, taking a $3 \sigma$ excess from the background level, we find:

$\frac{\beta^{2}}{2} \exp \left(-T P_{\mathrm{cpt}} / N \tau\right)<\frac{N_{3 \sigma}}{\epsilon_{\mathrm{tot}} P_{\mathrm{cpt}} N_{\mathrm{free}} N_{\mathrm{int}}}$.

In general, we can consider the case in which the target is composed of a number $v$ of electrically disconnected blocks (in our case $v=3$ ) each containing a number $N_{i}$ of atoms, $N_{\text {free }}^{i}$ conduction electrons and characterised by an age $T_{i}$, then the expected number of $X$ rays from violating PEP transitions is:

$$
\begin{aligned}
N_{X} & \approx \sum_{i=1}^{\nu} N_{\text {free }}^{i} N_{\text {int }} P_{\mathrm{cpt}} \epsilon_{\mathrm{tot}} \frac{\beta^{2}}{2} \exp \left(-T_{i} P_{\mathrm{cpt}} / N_{i} \tau\right) \\
& =N_{\text {int }} P_{\mathrm{cpt}} \epsilon_{\mathrm{tot}} \frac{\beta^{2}}{2} \sum_{i=1}^{\nu} N_{\text {free }}^{i} \exp \left(-T_{i} P_{\mathrm{cpt}} / N_{i} \tau\right),
\end{aligned}
$$

where the parameters $N_{\text {int }}$ and $P_{\text {cpt }}$ are common to all the $v$ blocks, and the detection efficiency $\epsilon_{\text {tot }}$ is also the same for all blocks in our experimental conditions. Accordingly, the experimental limit on the PEP violation probability generalises to:

$\frac{\beta^{2}}{2}<\frac{N_{3 \sigma}}{N_{\text {int }} P_{\mathrm{cpt}} \epsilon_{\mathrm{tot}} \sum_{i=1}^{v} N_{\text {free }}^{i} \exp \left(-T_{i} P_{\mathrm{cpt}} / N_{i} \tau\right)}$.

It is worth to stress that the order of magnitude of $N \tau_{S} / P_{c p t}$ is $10^{3}$ years for one mole of a lead target (even smaller for the interaction time $\tau_{E}$ ) as will be shown in Sects. 4.2 and 4.3. As a consequence, if the age $T\left(T_{i}\right)$ of the metal block ( $i$-th block) is of the order of a geologic time-scale (i.e. 

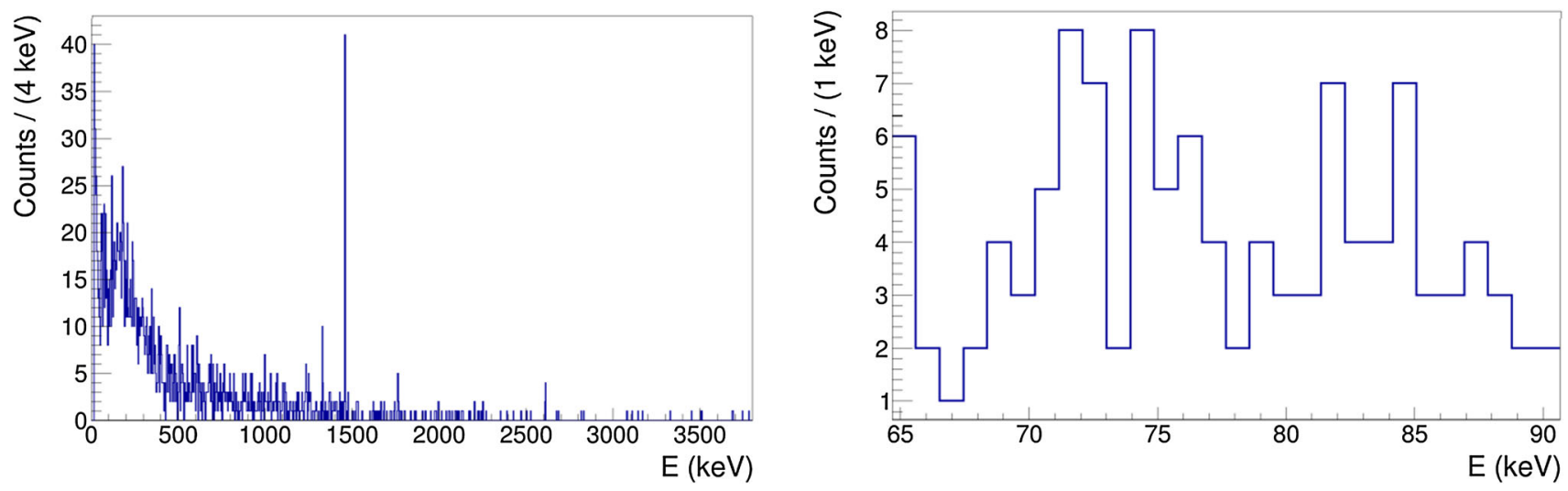

Fig. 2 Total measured $\mathrm{X}$-ray spectrum (left); same spectrum in the region of the $\mathrm{K}_{\alpha}$ standard and violating transitions in $\mathrm{Pb}$ (right)

it was not recently moulded from $n>1$ original lead fragments electrically disconnected) then each conduction electron of the target had a long time to interact with each atom of the target. Independently on the PEP violation model all the possible violating transitions must already have occurred at the time of the observation; whatever anomalous conduction electron-atom state, which existed at the formation of the metal block, the conduction electron must have already been captured and resides now in the K-shell of a stable nonPaulian atom. In this case the expected number of $X$ rays from violating PEP transitions, as given by Eq. (8) is $N_{X} \approx 0$. Let us now analyse in further detail the experimental condition of our measurement. Each of the $v=3$ blocks constituting our target were recently $\left(T_{i}=16\right.$ years before the measurement) realised in the same melting from a sample of $n>1$ original lead fragments. Each conduction electron of a given fragment had a long time to interact with each atom of the fragment and be eventually captured in a PEP violating atom. But, if the PEP violation is due to the wrong pairing of - well defined - electron pairs (as in Ref. [11]), an anomalous conduction electron of the fragment (among the $n$ ) may have his anomalous counterpart inside another fragment (among the remaining $n-1)$. Thus, after melting, all the electrons of a fragment can interact, and test, all the atoms belonging to the other $n-1$ fragments, searching for their anomalous counterparts. ${ }^{1}$ Equation (9) is to be modified accordingly:

$\frac{\beta^{2}}{2}<\frac{N_{3 \sigma}}{N_{\text {int }} P_{\text {cpt }} \epsilon_{\text {tot }} \sum_{i=1}^{v} N_{\text {free }}^{i} e^{-\frac{n}{n-1} \frac{T_{i} P_{\mathrm{cpt}}}{N_{i} \tau}}}$.

and the term $n /(n-1)$ accounts for the fact that all the atoms originally belonging to the melting fragment are not probes for our measurement. Stated differently, during the

\footnotetext{
1 The total number of atoms in the $i$-th lead block is $N_{i}=\sum_{j=1}^{n} N_{i}^{j}=n N_{i}^{j}$, assuming for the sake of simplicity the same number $N_{i}^{j}$ of atoms for each fragment.
}

time $T_{i}$ corresponding to the age of the $i$-th block after melting, each electron in the $i$-th block undergoes a mean number $T_{i} /\left(N_{i}-N_{i} / n\right) \tau$ of interactions with the atoms originally belonging to the other fragments. Obviously Eq. (10) reduces to Eq. (9) in the limit $n \rightarrow \infty$ of an infinite number of original fragments. Since we have no information concerning the original number $n$ of fragments which was used in the melting of each block of target, we adopt the most conservative assumption $n=2$ in what follows.

\subsection{Interactions as scatterings in the naive conduction model}

Let us first consider the picture in which electron-atom interactions correspond to the scatterings in the standard conduction picture. In this case we take $\tau=\tau_{S}$ with $\tau_{S} \approx$ $1.3 \times 10^{-15} \mathrm{~s}$ and $P_{\mathrm{cpt}}=0.009$ in lead (see Ref. [9]) then the time constant $N \tau / P_{\text {cpt }}$ in Eq. (7) evaluates to about 2800 years in the case of one mole of lead. The Roman lead blocks used in this experiment correspond to about 45, 42 and 21 moles respectively, this amplifies the time constant to about $12.4 \cdot 10^{4}, 11.5 \cdot 10^{4}$ and $5.8 \cdot 10^{4}$ years respectively. Because of these long time constants, with respect to the 16 years elapsed from the target blocks melting, each of the exponential factors in the denominator of Eq. (10) can be approximated to one, and $\sum_{i=1}^{v} N_{\text {free }}^{i}=N_{\text {free }}$ as given in Table 1.

Figure 2 shows the measured energy distribution. We note that the average value of the background counts in the range $\Delta E=65-90 \mathrm{keV}$, where the PEP violating $\mathrm{K}_{\alpha 1}$ and $\mathrm{K}_{\alpha 2}$ transitions are expected to occur, is $b=4.44$ counts $/ \mathrm{keV}$.

This is roughly four orders of magnitude lower than the background measured in Ref. [9]. We also notice that the standard $\mathrm{K}_{\alpha}$ lines are practically indistinguishable from the continuous background. This is partly due to the purity of the Roman lead target, and partly to the thickness of the target which is of the order of $5 \mathrm{~cm}$, much thicker than the 
target used in [9] which was about $0.1 \mathrm{~cm}$ thick. The target contributes to suppress the residual background eventually surviving the external lead and copper shielding layers. The photo-absorption length in $\mathrm{Pb}$ at the energies of the $\mathrm{K}_{\alpha 1}$ and $\mathrm{K}_{\alpha 2}$ transitions (i.e. in the energy range $71-74 \mathrm{keV}$ ) is of about $350 \mu \mathrm{m}$. Due to self-absorptions only the inner target shell contributes to the efficiency, the rest of the target acting as passive shielding.

The precise positions of the two main lines $\mathrm{K}_{\alpha 1}$ and $\mathrm{K}_{\alpha 2}$ in $\mathrm{Pb}$ are reported in Table 2 together with the energies of the corresponding PEP violating transitions. The energies of these transitions were calculated with an estimated accuracy of few eV. These values are obtained on the basis of a DiracHartree-Slater calculation that includes the Breit interaction and QED corrections (we refer the reader to Ref. [9] for more details and further references concerning the calculation).

Since the violating $\mathrm{K}_{\alpha}$ transitions are close to the corresponding normal lines, taking into account the intrinsic resolution of the detector which is of the same order, in Ref. [9] two optimal Regions Of Interest (ROI) around the forbidden transition energies are defined. For an easier comparison of our results with those presented in [9] we adopt the same ROI ranges and $\epsilon_{R O I}$. This is also motivated by the fact that in our spectrum the $\mathrm{K}_{\alpha}$ lines barely emerge - if at all - over the low continuous background and a similar minimisation would make little sense.

Table 3 lists the ROI intervals for the violating $\mathrm{K}_{\alpha}$ lines, together with the corresponding efficiency factors and the total efficiency $\left(\epsilon_{t o t}\right): \epsilon_{B R}$ denotes the branching fraction for the $\mathrm{K}_{\alpha}$ line, and $\epsilon_{x}$ is the detection efficiency, for photons emitted inside the $\mathrm{Pb}$ target, at the energy corresponding to the violating $\mathrm{K}_{\alpha}$ atomic transition. In Table 3 the expected number of counts (given the average $b$ ) and the observed $\mathrm{X}$-ray counts in the two ROI regions are also reported.

The fluctuations in both ROI regions have a Poisson statistics and the likelihood (the probability distribution of the data) evaluated in the case of null hypothesis is:

$\mathcal{L}\left(z_{1}, z_{2}\right)=\frac{b^{z_{1}}}{z_{1} !} \exp (-b) \frac{b^{z_{2}}}{z_{2} !} \exp (-b)$

where $z_{1}$ and $z_{2}$ are, respectively, the measured counts in the first and in the second ROI. Then the probability of obtaining

Table $2 \mathrm{~K}_{\alpha}$ atomic transition energies in $\mathrm{Pb}$ resulting from violation of the Pauli Exclusion Principle, indicated by the column labeled (forb.). For reference the allowed transition energies are also quoted (allow.). Energies are in eV. See Ref. [9] for details

\begin{tabular}{lll}
\hline Transitions in $\mathrm{Pb}$ & Forb. & Allow. \\
\hline $1 \mathrm{~s}-2 \mathrm{p}_{3 / 2} \mathrm{~K}_{\alpha 1}$ & 73713 & 74961 \\
$1 \mathrm{~s}-2 \mathrm{p}_{1 / 2} \mathrm{~K}_{\alpha 2}$ & 71652 & 72798 \\
\hline
\end{tabular}

counts that are higher than those actually measured in both ROI's is given by the expression

$$
\begin{aligned}
p & =\sum_{j=z_{1}+1}^{\infty} \frac{b^{j}}{j !} \exp (-b) \sum_{k=z_{2}+1}^{\infty} \frac{b^{k}}{k !} \exp (-b) \\
& =\left[1-\sum_{j=0}^{z_{1}} \frac{b^{j}}{j !} \exp (-b)\right]\left[1-\sum_{k=0}^{z_{2}} \frac{b^{k}}{k !} \exp (-b)\right],
\end{aligned}
$$

which evaluates to 0.012 with the measured counts $z_{1}=1$ and $z_{2}=7$. This is a $p$ value that corresponds to 2.3 standard deviations (one-sided $\mathrm{p}$ value test), which is far too low to claim that the null hypothesis has been invalidated by the data and that there is a violation of the Pauli Exclusion Principle.

A $3 \sigma$ upper limit on the PEP violation probability $\beta^{2} / 2$ can be obtained from Eq. (10) with the approximation $\sum_{i=1}^{v} N_{\text {free }}^{i} e^{-\frac{n}{n-1} \frac{T_{i} P_{\text {cpt }}}{N_{i} \tau}} \approx N_{\text {free }}$ :

$\frac{\beta^{2}}{2}<\frac{N_{3 \sigma}}{\epsilon_{\text {tot }} P_{\text {cpt }} N_{\text {free }} N_{\text {int }}}$,

where $N_{3 \sigma}$ is the estimate of a $3 \sigma$ deviation from the Poisson statistics of the assumed flat background in the energy region 65-90 keV. The two lines also differ in total efficiency and therefore our estimate of the $3 \sigma$ deviation to be used in Eq. (13) is given by:

$$
\frac{N_{3 \sigma}}{\epsilon_{\mathrm{tot}}}=3 \sqrt{\frac{z_{1}}{\epsilon_{1, t o t}^{2}}+\frac{z_{2}}{\epsilon_{2, t o t}^{2}}} .
$$

In Eq. (14) $z_{1}=1$ and $z_{2}=7$ are again the number of counts in the two ROIs, while $\epsilon_{1, \text { tot }}$ and $\epsilon_{2, \text { tot }}$ are the corresponding total efficiencies. By substituting Eq. (14) and the values reported in Table 1 and Table 3 into the bound Eq. (13) the $3 \sigma$ upper limit on the PEP violation probability is found to be:

$\frac{1}{2} \beta^{2}<1.58 \cdot 10^{-40}$

which improves the result obtained in [9] by a factor 16 .

\subsection{Interactions as "close encounters"}

In the new framework outlined in Ref. [10] we use the closest approaches to atoms instead of scatterings, corresponding to a much shorter $\tau=\tau_{E}=2.5 \times 10^{-17} \mathrm{~s}$ in lead. In this scheme the interaction always gives rise to absorption, hence 
Table 3 The ROI intervals for the forbidden $\mathrm{K}_{\alpha}$ atomic transitions, the corresponding efficiency factors, the expected number of counts with the average value of $b=4.44$ counts/keV and the observed $\mathrm{X}$-ray counts in the same regions

\begin{tabular}{llllllll}
\hline Forb. transitions & ROI & $\epsilon_{R O I}$ & $\epsilon_{B R}$ & $\epsilon_{x}$ & $\epsilon_{\text {tot }}$ & Expected & Counts \\
\hline $\mathrm{K}_{\alpha 1}$ & $(73.4 \div 74.1) \mathrm{keV}$ & 0.811 & 0.47 & $5.0 \cdot 10^{-5}$ & $1.9 \cdot 10^{-5}$ & 3.1 & 1 \\
$\mathrm{~K}_{\alpha 2}$ & $(71.3 \div 72.0) \mathrm{keV}$ & 0.834 & 0.23 & $3.6 \cdot 10^{-5}$ & $7.0 \cdot 10^{-6}$ & 3.1 & 7 \\
\hline
\end{tabular}

the probability for PEP violation assumes the expression:

$$
\frac{\beta^{2}}{2}<\frac{N_{3 \sigma}}{N_{\text {int }} \epsilon_{\text {tot }} \sum_{i=1}^{v} N_{\text {free }}^{i} e^{-\frac{n}{n-1} \frac{T_{i}}{N_{i} \tau}}} .
$$

In order to extract a $3 \sigma$ bound we substitute $N_{X}^{m}=N_{3 \sigma}$ in Eq. (16). By substituting $n=2$ the sum in the denominator of Eq. (16) amounts to:

$\sum_{i=1}^{v} N_{\text {free }}^{i} e^{-\frac{n}{n-1} \frac{T_{i}}{N_{i} \tau}} \sim 5.19 \cdot 10^{25}$.

By applying Eq. (16) the limit on the PEP violation probability then turns to be:

$\frac{1}{2} \beta^{2}<1.53 \cdot 10^{-43}$,

which improves the result in [9] by more then four orders of magnitude.

\section{Conclusions and perspectives}

The ancient Roman lead is an important resource for lowbackground experiments, and here we have used it to obtain an exceptionally low noise measurement.

The bounds (15) and (18), corresponding to the two electron-atom interaction schemes, represent the best limits measured on the PEP violation probability for electrons, provided that a superselection rule prevents transitions between normal and anomalous states in a closed system and the violation is justified by assuming that the global wave function of all the electrons in the universe contains a number of "wrong" signs like in Ref. [11].

In the future it may be interesting to produce a new block of Roman lead, by melting a large number $n$ of Roman lead fragments, in order to further improve the limits on the probability of PEP violation for electrons with this kind of test.

Acknowledgements We acknowledge the support of the Centro Fermi - Museo Storico della Fisica e Centro Studi e Ricerche "Enrico Fermi" (Open Problems in Quantum Mechanics project), the support from the EU COST Action CA 15220 is gratefully acknowledged. We acknowledge the support from the H2020 FET Project TEQ (Grant no. 766900).
We thank the Austrian Science Foundation (FWF) which supports the VIP2 project with the grants P25529-N20, project P 30635-N36 and W1252-N27 (doctoral college particles and interactions).

Data Availability Statement This manuscript has no associated data in a data repository. [Authors' comment: Source data that support the plots within this paper and other findings of this study are available from the corresponding author upon reasonable request.]

Open Access This article is licensed under a Creative Commons Attribution 4.0 International License, which permits use, sharing, adaptation, distribution and reproduction in any medium or format, as long as you give appropriate credit to the original author(s) and the source, provide a link to the Creative Commons licence, and indicate if changes were made. The images or other third party material in this article are included in the article's Creative Commons licence, unless indicated otherwise in a credit line to the material. If material is not included in the article's Creative Commons licence and your intended use is not permitted by statutory regulation or exceeds the permitted use, you will need to obtain permission directly from the copyright holder. To view a copy of this licence, visit http://creativecomm ons.org/licenses/by/4.0/.

Funded by $\mathrm{SCOAP}^{3}$.

\section{References}

1. M. Fierz, Über die relativistische Theorie kräftefreier Teilchen mit beliebigem Spin. Helvetica Phys. Acta 12, 12 (1939)

2. W. Pauli, The connection between spin and statistics. Phys. Rev. 58(8), $716(1940)$

3. O.W. Greenberg, A.M.L. Messiah, Symmetrization postulate and its experimental foundation. Phys. Rev. B 136, 248-67 (1964)

4. E. Ramberg, G.A. Snow, Experimental limit on a small violation of the pauli principle. Phys. Lett. B 238(2), 438-441 (1990)

5. O.W. Greenberg, R.N. Mohapatra, Local quantum field theory of possible violation of the pauli principle. Phys. Rev. Lett. 59(22), 2507-2510 (1987)

6. C. Curceanu Petrascu et al., Experimental tests of quantum mechanics-Pauli exclusion principle violation (the VIP experiment) and future perspective. J. Phys. Conf. Ser. 306, 012036 (2011)

7. C. Curceanu Petrascu et al., Experimental tests of quantum mechanics-Pauli exclusion principle violation (the VIP experiment) and future perspective. Phys. Procedia 17, 40 (2011)

8. H. Shi, E. Milotti, S. Bartalucci, M. Bazzi, S. Bertolucci, A.M. Bragadireanu, M. Cargnelli, A. Clozza, L. De Paolis, S. Di Matteo, J.-P. Egger, H. Elnaggar, C. Guaraldo, M. Iliescu, M. Laubenstein, J. Marton, M. Miliucci, A. Pichler, D. Pietreanu, K. Piscicchia, A. Scordo, D.L. Sirghi, F. Sirghi, L. Sperandio, O. Vazquez Doce, E. Widmann, J. Zmeskal, C. Curceanu, Experimental search for the violation of pauli exclusion principle. Eur. Phys. J. C 78(4), 319 (2018) 
9. S.R. Elliott, B.H. LaRoque, V.M. Gehman, M.F. Kidd, M. Chen, An improved limit on pauli-exclusion-principle forbidden atomic transitions. Found. Phys. 42(8), 1015-1030 (2012)

10. E. Milotti, S. Bartalucci, S. Bertolucci, M. Bazzi, M. Bragadireanu, M. Cargnelli, A. Clozza, C. Curceanu, L. De Paolis, J.-P. Egger et al., On the importance of electron diffusion in a bulk-matter test of the pauli exclusion principle. Entropy 20(7), 515 (2018)

11. V. Rahal, A. Campa, Thermodynamical implications of a violation of the pauli principle. Phys. Rev. A 38(7), 3728-3731 (1988)

12. C. Kittel, Introduction to Solid State Physics, 7th edn. (Wiley, New York, 2007)

13. A. Alessandrello, C. Arpesella, C. Brofferio, C. Bucci, C. Cattadori, O. Cremonesi, A. Ettore Fiorini, S.Latorre Giuliani, A. Nucciotti et al., Measurements of internal radioactive contamination in samples of roman lead to be used in experiments on rare events. Nucl. Instr. Methods Phys. Res. Sect. B Beam Interact. Mater. Atoms 142(1-2), 163-172 (1998)

14. N. Nosengo, Roman ingots to shield particle detector. Nature 2010, 24 (2010)

15. H. Neder, G. Heusser, M. Laubenstein, Low level $\gamma$-ray germanium-spectrometer to measure very low primordial radionuclide concentrations. Appl. Radiat. Isotopes 53(1-2), 191-195 (2000)

16. G. Heusser, M. Laubenstein, H. Neder, Low-level germanium gamma-ray spectrometry at the $\mu \mathrm{bq} / \mathrm{kg}$ level and future developments towards higher sensitivity. Radioactivity Env. 8, 495-510 (2006)

17. F.J. Dyson, A. Lenard, Stability of matter. I. J. Math. Phys. 8(3), 423-434 (1967)

18. A. Lenard, F.J. Dyson, Stability of matter. II. J. Math. Phys. 9(5), 698-711 (1968)

19. E. Fermi, Le ultime particelle costitutive della materia. Scientia 28(55), 21 (1934)

20. E. Milotti, Enrico Fermi's view of identical particles (2007)

21. R.D. Amado, H. Primakoff, Comments on testing the Pauli principle. Phys. Rev. C 22, 1338-1340 (1980)

22. G.J. Gentile, Itosservazioni sopra le statistiche intermedie. Il Nuovo Cimento (1924-1942) 17, 493-497 (1940)
23. O.W. Greenberg, Interactions of particles having small violations of statistics. Phys. A Stat. Mech. Appl. 180(3-4), 419-427 (1992)

24. H.S. Green, A generalized method of field quantization. Phys. Rev. 90(2), 270 (1953)

25. G.F. Dell'Antonio, O.W. Greenberg, E.C.G. Sudarshan. Group theoretical concepts and methods in elementary particle physics. In F. Gürsey-NY ed. Lectures at the Istanbul Summer School of Theoretical Physics (1962). Gordon and Breach, pp. 403 (1964)

26. A.Y. Ignatiev, V.A. Kuzmin, Is small violation of the Pauli principle possible? In A.N. Tavkhelidze, V.A. Matveev, A.A. Pivovarov, I.I. Tkachev, eds. Quarks '86: Proceedings of the Seminar, Tbilisi, USSR, 15-17 April 1986

27. A.Y. Ignatiev, $X$ rays test the Pauli exclusion principle. Proceedings of the 20th International Conference on X-ray and Inner-Shell Processes. Radiat. Phys. Chem. 75(11), 2090-2096 (2006)

28. L.B. Okun, Possible violation of the pauli principle in atoms. JETP Lett. 46, 11 (1987)

29. A.B. Govorkov, Can the Pauli principle be deduced with local quantum field theory? Phys. Lett. A 137(1-2), 7-10 (1989)

30. O.W. Greenberg, Example of infinite statistics. Phys. Rev. Lett. 64, 705 (1990)

31. A.B. Govorkov, The existence of antiparticles seems to forbid violations of statistics. Phys. A Statist. Mech. Appl. 203(3), 655-670 (1994)

32. M. Boswell, Y.-D. Chan, J.A. Detwiler, P. Finnerty, R. Henning, V.M. Gehman, R.A. Johnson, D.V. Jordan, K. Kazkaz, M. Knapp et al., Mage-a geant4-based monte carlo application framework for low-background germanium experiments. IEEE Trans. Nucl. Sci. 58(3), 1212-1220 (2011)

33. S. Agostinelli, K. John Allison, A. Amako, J. Apostolakis, H. Araujo, P. Arce, M. Asai, D. Axen, S. Banerjee, G. Barrand et al., GEANT4 - a simulation toolkit. Nuclear Instr. Methods Phys. Res. Sect. A Acceler. Spectrometers Detect. Assoc. Equip. 506(3), 250 303 (2003) 